\title{
Electroacupuncture remediates glial dysfunction and ameliorates neurodegeneration in the astrocytic a-synuclein mutant mouse model
}

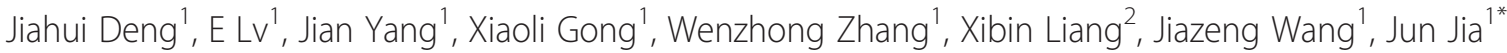 \\ and Xiaomin Wang ${ }^{1 *}$
}

\begin{abstract}
Background: The acupuncture or electroacupuncture (EA) shows the therapeutic effect on various neurodegenerative diseases. This effect was thought to be partially achieved by its ability to alleviate existing neuroinflammation and glial dysfunction. In this study, we systematically investigated the effect of EA on abnormal neurochemical changes and motor symptoms in a mouse neurodegenerative disease model.

Methods: The transgenic mouse which expresses a mutant a-synuclein (a-syn) protein, A53T a-syn, in brain astrocytic cells was used. These mice exhibit extensive neuroinflammatory and motor phenotypes of neurodegenerative disorders. In this study, the effects of EA on these phenotypic changes were examined in these mice.

Results: EA improved the movement detected in multiple motor tests in A53T mutant mice. At the cellular level, EA significantly reduced the activation of microglia and prevented the loss of dopaminergic neurons in the midbrain and motor neurons in the spinal cord. At the molecular level, EA suppressed the abnormal elevation of proinflammatory factors (tumor necrosis factor- $a$ and interleukin-1 $\beta$ ) in the striatum and midbrain of A53T mice. In contrast, EA increased striatal and midbrain expression of a transcription factor, nuclear factor E2-related factor 2 , and its downstream antioxidants (heme oxygenase-1 and glutamate-cysteine ligase modifier subunits).

Conclusions: These results suggest that EA possesses the ability to ameliorate mutant a-syn-induced motor abnormalities. This ability may be due to that EA enhances both anti-inflammatory and antioxidant activities and suppresses aberrant glial activation in the diseased sites of brains.
\end{abstract}

Keywords: Astrogliosis, a-synuclein, Electroacupuncture, Glial dysfunction, Nrf2

\section{Background}

As a conventional cytosolic protein, $\alpha$-synuclein ( $\alpha$-syn), has been assumed to exert its pathogenic function exclusively in the cytoplasm in a cell-autonomous manner [1]. However, this view has been challenged by the presence of $\alpha$-syn and its aggregated forms in the extracellular fluid such as cerebrospinal fluid and blood plasma of both Parkinson's disease (PD) and normal subjects [2, 3]. Although the mechanisms underlying $\alpha$-syn secretion are not fully understood, evidence has shown that $\alpha$-syn

\footnotetext{
* Correspondence: jiajun@ccmu.edu.cn; xmwang@ccmu.edu.cn

'Departments of Neurobiology and Physiology, Key Laboratory for

Neurodegenerative Disorders of the Ministry of Education, Beijing Key

Laboratory for Parkinson's Disease, Capital Medical University; Beijing Institute

for Brain Disorders, Beijing 100069, China

Full list of author information is available at the end of the article
}

fibrils are released to the extracellular environment by non-classical exocytosis $[4,5]$. Extracellularly misfolded $\alpha$ syn can propagate in a prion-like spreading fashion $[6,7]$ and be endocytosed by neighboring neurons or glia [8], through which $\alpha$-syn spreads and amplifies degenerative signals from cells to cells [9].

Glial cells are particularly involved in cell-to-cell contacts of $\alpha$-synucleinopathies in the progression of neurodegenerative disorders [10]. Several lines of evidence have shown that $\alpha$-syn-containing inclusion bodies aggregated in astrocytes and oligodendrocytes in PD, dementia with lewy bodies, and multiple system atrophy [11-13]. In fact, $\alpha$-syn-positive inclusions have been observed in astrocytes and oligodendrocytes in the substantia nigra (SN) and/or locus coeruleus of postmortem 
PD patients, and the number of glial $\alpha$-syn-inclusions was related to the progress of PD [14]. However, the underlying mechanisms for $\alpha$-synucleinopathies in glia and its roles in disease onset and progression remain unclear.

An experimental $\alpha$-syn-inducible transgenic mouse, in which PD-related A53T mutant $\alpha$-syn is selectively expressed in astrocytes, developed an early-onset, rapidly progressed movement disability. Increasing astrocytic $\alpha$ syn deposition initiated widespread astrogliosis, microglial activation, and dopaminergic neuronal degeneration [15]. It appears that $\alpha$-syn induces early astrocytic dysfunction and severe inflammation responses, which accelerate neuronal damage and PD progression [10]. In addition, $\alpha$-syn is released from neuronal cells, and the released $\alpha$-syn is readily endocytosed by astrocytes, leading to neuroinflammatory responses [16]. Therefore, restoring glial function and inhibiting microglial activation are of therapeutic benefit for treating PD [17].

Due to severe side effects induced by long-term medications for treating chronic neurodegenerative diseases, it is necessary to find non-pharmacological tools to ameliorate these disorders. Acupuncture or electroacupuncture (EA) represents a non-invasive procedure and has long been used to relieve pain and promote stroke rehabilitation $[18,19]$. Several neurodegenerative disorders, such as amyotrophic lateral sclerosis [20], ischemia [21], and PD [22, 23], are also seemingly suitable for EA treatment. Although the use of EA has been endorsed by the US National Institutes of Health and the World Health Organization, and the anti-inflammatory effect of acupuncture has been well documented in human and animal studies [24-27], little is understood about its biochemical basis for therapy.

In this study, we attempted to investigate the neurochemical basis for the therapeutic effect of EA. To this end, we used a transgenic mouse line which was constructed to selectively express $\alpha$-syn A53T mutant in astrocytic cells. The anti-inflammatory effect of EA was investigated in these mice. The effects of EA on motor behavior and activities of glial and neuronal cells were also examined. In addition, the nuclear factor E2-related factor 2 (Nrf2)-antioxidant response element (ARE) signaling pathway, a key mediator in oxidative stress and inflammation [28], was analyzed for its responses to EA.

\section{Methods}

\section{Animals}

TetO-A53T mice, tetracycline operator (tetO)-regulated $\alpha$-syn transgenic mice, and glial fibrillary acidic protein (GFAP)-tTA mice (GFAP promoter-controlled tetracycline transactivator transgenic mice) were obtained from Prof. Huaibin Cai's laboratory in the National Institutes of Health. These mice were crossed to generate GFAP-
tTA/tetO- $\alpha$-syn (A53T) double transgenic mice. The mice were housed in a pathogen-free climate-controlled facility with diet and water available ad libitum. The animal room was on a 12/12 h light/dark cycle. To assess the impact of developmental expression of A53T $\alpha$-syn on the neurological function, a cohort of A53T mice was administered with doxycycline (DOX), a tetracycline derivative, to block the expression of A53T $\alpha$-syn from the embryonic stage to postnatal day 21 (P21). Genotypes were determined by polymerase chain reaction (PCR) analysis of tail DNA [15]. All procedures were approved by the Ethical Committee for Animal Research of Capital Medical University.

\section{EA stimulation}

Mice were divided into four groups: untreated nontransgenic ( $\mathrm{nTg}$ ) control, untreated A53T transgenic, $100 \mathrm{~Hz}$ EA-treated nTg, and $100 \mathrm{~Hz}$ EA-treated A53T mice. The EA stimulation was given for 4 weeks starting at 2 months of age. Mice were gently handled and lightly restrained in a plastic cylinder $(7 \times 2.5 \mathrm{~cm})$ with their hind limbs accessible for needling. For this procedure, the mice were immobilized for 30 min during EA administration with no anesthetization [29]. After cleaning the skin with alcohol, two sterilized stainless-steel needles $(0.18 \mathrm{~mm}$ diameter $\times 3 \mathrm{~mm}$ length) were inserted in each leg, one at ST36 (2 mm lateral to the anterior tubercle of tibia) and the other at SP6 (2 mm proximal to the upper border of medial malleolus, at the posterior border of the tibia). The EA performed at these acupoints is known to relieve PDlike symptoms such as muscle and movement disorders [30]. The stimuli were generated from a Han's Acupoint Nerve Stimulator (HANS, LH series, manufactured in Peking University). Bidirectional square wave electrical pulses $(0.2 \mathrm{~ms}$ duration, $100 \mathrm{~Hz})$ designated as EA were given for a total of $30 \mathrm{~min}$ each day. The intensity of the stimulation at $100 \mathrm{~Hz}$ was increased stepwise from 1 to $1.2 \mathrm{~mA}$ and then to $1.4 \mathrm{~mA}$, with each step lasting for $10 \mathrm{~min}$.

\section{Behavioral test \\ Open-field test}

Locomotor activity was assessed in automated activity chambers $(25.4 \times 25.4$ in. square $)$ connected to a digital scan analyzer that transmitted the number of infrared beam breaks (activity data) to a Mouse Tru Scan system (Truscan 2.0 Instruments, Columbus, USA). Total movement distance $(\mathrm{cm})$ was recorded across a 30-min recording period.

\section{Rotarod test}

The length of time a mouse stayed on a rotating rod with auto acceleration from 0 rotation per minute (rpm) to 40 rpm in 5 min (Acceler ROTA-ROD, Jones \& Roberts, CA) was recorded. 


\section{Grip strength measurement}

Mouse forepaws or hindpaws were pulled or compressed a triangular bar linked to a digital force gauge (San Diego Instruments, San Diego, CA). The maximal pulling or compressing force was recorded. The mean of five measurements was analyzed for each animal during each test.

\section{Gait analysis}

The Catwalk gait analysis method has been reported in details [31]. Briefly, mice were placed individually in the Catwalk walkway system (Noldus Information Technology, The Netherlands) and were allowed to walk freely and traverse from one side to the other of the walkway glass plate. During the recording, the environment was kept completely dark, except for the light from the computer screen. Where the mouse paws made contact with the glass plate, light emitting diode light was reflected down and the illuminated contact areas were recorded with a high speed color video camera underneath the glass plate connected to a computer that run the Catwalk software 9.1. Stride length and interlimb coordination measured as regularity index, which is the percentage of normal step sequences, were analyzed.

\section{Histology and immunohistochemical analysis}

Mice were deeply anesthetized and transcardially perfused with saline followed by fixative (4\% paraformaldehyde in $0.1 \mathrm{M}$ phosphate buffered saline (PBS), pH 7.4. Brain and spinal cord tissues were removed and postfixed in the same fixative overnight. Following equilibration in $30 \%$ sucrose in PBS, $30 \mu \mathrm{m}$ sections were cut in the coronal plane with a cryostat at $-20{ }^{\circ} \mathrm{C}$ and processed for immunohistochemistry. Primary antibodies against GFAP (1:1000, Sigma-Aldrich USA, St. Louis, $\mathrm{MO}$ ), ionized calcium-binding adaptor molecule-1 (Iba1) (1:1000, Wako Chemicals USA, Richmond, VA), tyrosine hydroxylase (TH) (1:1000, Sigma-Aldrich USA, St. Louis, MO), $\alpha$-syn (C20 \& 211, 1:1000, Santa Cruz Biotech, Santa Cruz, CA), and secondary Alexa 488- or Alex 568conjugated antibodies (1:1000, Invitrogen) were used to visualize the staining. Dapi (1:1000, Invitrogen) was used for counterstaining the nuclei. A monoclonal mouse anti-neuronal Nuclei (NeuN) antibody was used at a 1:1000 dilution (Chemicon International, Temecula, CA). Immunofluorescent images were captured using a laser scanning confocal microscope (Leica TCS SP8, Germany).

\section{Stereology}

According to stereotaxic coordinates of mouse brains [32], a series of coronal sections across the substantia nigra pars compacta (SNpc) (Bregma $-2.54 \sim-3.88 \mathrm{~mm}$ ) was stained with $\mathrm{TH}$ and visualized using the Vectastain $\mathrm{ABC}$ kit (Vector Laboratories, Burlingame, CA). The number of TH-positive cells was assessed using an unbiased stereological procedure with an optical fractionator Stereo Investigator (Micro-Bright Field Inc, Williston, $\mathrm{VT})$. The coefficient of error (CE) was designed less than $10 \%$ in order to get reliable results.

\section{Quantitation of proinflammatory cytokines}

We quantified proinflammatory cytokine levels in the $\mathrm{SNpc}$ and striatum. Tissues were homogenized in ice-cold lysis buffer containing $137 \mathrm{mM} \mathrm{NaCl}, 20 \mathrm{mM}$ Tris (pH 8.0), $1 \%(v / v)$ glycerol, $1 \%(v / v)$ Nonidet P- $40,1 \mathrm{mM}$ phenylmethylsulfonyl fluoride, and $0.5 \mathrm{mM}$ sodium vanadate. The homogenate was centrifuged at $1500 \mathrm{~g}$ for $15 \mathrm{~min}$ at $4{ }^{\circ} \mathrm{C}$. The supernatant was collected and stored at $-80{ }^{\circ} \mathrm{C}$ before use. The levels of tumor necrosis factor- $\alpha$ (TNF- $\alpha$ ) and interleukin-1 $\beta$ (IL-1 $\beta$ ) were detected using mouse TNF- $\alpha$ and IL- $1 \beta$ enzyme-linked immunosorbent assay (ELISA) kits (Shanghai ExCell Biology Inc., Shanghai, China), respectively, according to the manufacturer's instructions. The sensitivity of the ELISA assay was 15 $\mathrm{pg} / \mathrm{ml}$ for both TNF- $\alpha$ and IL-1 $\beta$. Standards were assayed in duplicates. The protein concentration was determined using a detergent compatible protein assay with bovine serum albumin as the standard.

\section{Western blot}

Proteins were separated on $10 \%$ SDS-PAGE gels and electrophoretically transferred to polyvinylidene difluoride membranes (Millipore). Membranes were blocked with $5 \%$ nonfat dry milk in PBS for $2 \mathrm{~h}$ at room temperature and incubated overnight at $4{ }^{\circ} \mathrm{C}$ with a primary antibody. Antibodies against $\alpha$-syn (1:1000, Santa Cruz Biotech, Santa Cruz, CA), Nrf2 (1:500, Sigma-Aldrich USA, St. Louis, MO), heme oxygenase-1 (HO-1) (1:300, Abcam, Cambridge, UK), glutamate-cysteine ligase modifier subunits (GCLM) (1:500, Abcam, Cambridge, UK), or $\beta$-actin (1:5000, Sigma-Aldrich USA, St. Louis, $\mathrm{MO}$ ) were used in this study. The membranes were then treated with an anti-rabbit or anti-mouse antibody conjugated with IRDye ${ }^{\text {Tx }} 800$ green or IRDye ${ }^{\text {Tx }} 700$ red (Rockland, Limerick, PA) for $1 \mathrm{~h}$. They were washed three times with PBS containing $0.1 \%$ Tween and twice with PBS alone. Proteins were visualized by a LI-COR Odyssey infrared double-fluorescence imaging system (American Company LICOR).

\section{High-performance liquid chromatography (HPLC)}

Samples from striatal tissue were prepared to detect the DA concentration as previously described [29]. Each sample was injected into an HPLC system (Model 5600A; CoulArray Detector System ESA, Brighton, MA) for analysis. The mobile phase was $0.125 \mathrm{M}$ sodium citrate buffer containing $20 \%$ methanol, $0.1 \mathrm{mM} \mathrm{Na}{ }_{2}$ EDTA, 
and $0.5 \mathrm{mM}$ 1-octanesulfonic acid (Acros Organics, Morris Plains, NJ).

\section{Isolation of total RNA and quantitative real-time PCR}

The isolation of total RNA was performed by using an RNA NucleoSpin ${ }^{\circ}$ kit according to the manufacturer's instructions (Macherey-Nagel, Germany). Reverse transcriptase reactions were run on $1 \mu \mathrm{g}$ of total messenger RNA (mRNA) by using ProtoScript M-Mulv First Strand cDNA Synthesis Kit (New England Biolabs, USA). Quantitative PCR was performed by using a Brilliant IISYBR Green QPCR Master Mix (Agilent Technologies, USA). The primers sequences were $\alpha$-synF $\left(5^{\prime}\right.$-TGG ATG TAT TCA TGA AAG GA-3'), $\alpha$-synR (5'-CCA GTG GCT GCT GCA ATG CTC-3'), GFAPF (5'-CGA GTC CCT AGA GCG GCA AAT G-3'), GFAPR (5'-CGG ATC TGG AGG TTG GAG AAA GTC-3'), Nrf2/5' (5'-TTC TTT CAG CAG CAT CCT CTC CAC-3'), Nrf2/3' (5'ACA GCC TTC AAT AGT CCC GTC CAG-3'), HO-1/ 5' (5' -CAA GCC GAG AAT GCT GAG TTC ATG-3'), HO-1/3' (5'-GCA AGG GAT GAT TTC CTG CCA G-3'), GCLM/5' (5'-GCC ACC AGA TTT GAC TGC CTT TG-3'), GCLM/3' (5'-TGC TCT TCA CGA TGA CCG AGT ACC-3'), ActinF (5'-CTG GCT CCT AGC ACC ATG AAG ATC-3'), ActinR (5'-TGC TGA TCC ACA TCT GCT GG-3').

\section{Statistical analysis}

All data are represented as means \pm SEM and were analyzed by one-way ANOVA or Student's $t$ test as appropriate by using Prism 5.0 software (GraphPad Software, San Diego, CA). $P<0.05$ was considered statistically significant.

\section{Results}

EA delayed the early onset of movement disability in A53T mice

A53T $\alpha$-syn mice were administered with DOX from embryonic stages (E0) to P21 to block the developmental expression of A53T $\alpha$-syn (Fig. 1a). At day 60 (P60), A53T or nTg control mice received 4-week EA treatment. Before and after EA treatment, motor behaviors were examined.

A53T mice displayed a loss of body weight as compared to age-matched nTg mice at 70th day. The body weight of A53T mice continued to drop during the disease progression. EA at $100 \mathrm{~Hz}$ significantly prevented the loss of body weight. However, EA had no effect on the body weight of nTg mice (Fig. 1b). The early-onset paralysis was also seen in A53T mice which may result from the significant neuronal loss in the midbrain and spinal cord [15]. Average age of onset for paralysis developed with one of four limbs in A53T mice was $92.9 \pm$ 2.3 days. This symptom then quickly spread to other limbs which eventually caused death of animals at an average lifespan of $99.4 \pm 2.0$ days (Fig. 1c, d). Interestingly, $100 \mathrm{~Hz}$ EA significantly delayed the average age of onset to $102.7 \pm 2.1$ days and prolonged the average lifespan to $108.9 \pm 2.3$ days (Fig. 1c, d).

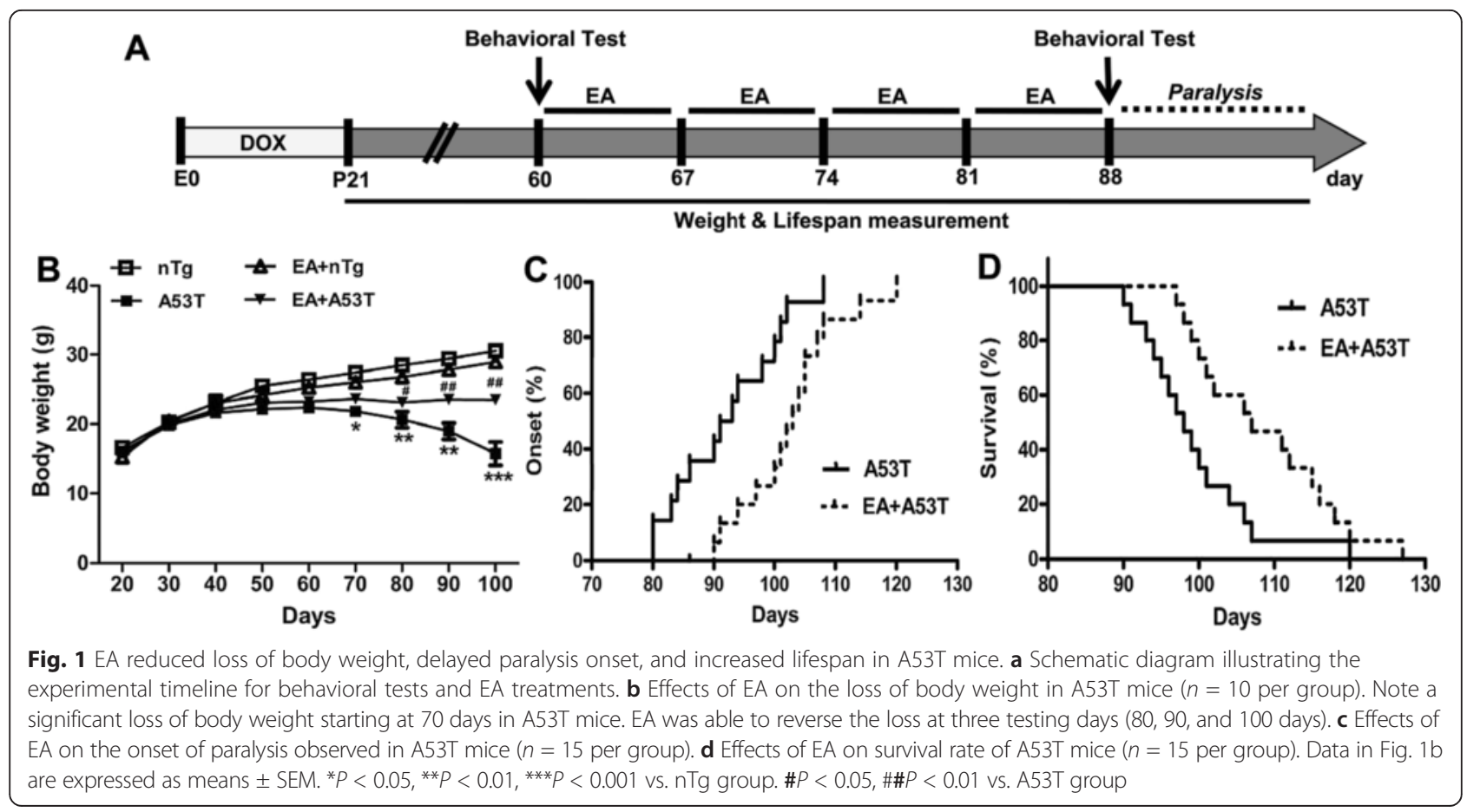


To examine the effect of EA on motor behaviors, openfield test was first carried out to assess the spontaneous locomotor activity in A53T and nTg mice after 4-week EA treatment. The movement distance was significantly decreased in A53T mice $(2221.7 \pm 160.0 \mathrm{~cm})$ compared to $\mathrm{nTg}$ mice $(4145.9 \pm 252.3 \mathrm{~cm})$. EA at $100 \mathrm{~Hz}$ effectively increased the movement distance $(3320.1 \pm 211.0 \mathrm{~cm})$ (Fig. 2a). The motor coordination of A53T mice was decreased in a rotarod test, which was partially reversed by $100 \mathrm{~Hz}$ EA (Fig. 2b). The grip strength test is a feasible way to objectively quantify the muscular strength of rodents. The mean grip force in the fore- and hindlimbs of A53T mice was significantly reduced relative to $\mathrm{nTg}$ mice (Fig. 2c). EA (4 weeks, $100 \mathrm{~Hz}$ ) restored the mean grip force by 62.9 and $35.6 \%$ respectively in the fore- and hindlimbs compared to A53T mice without EA (Fig. 2c). In addition, Catwalk gait analysis showed a significant decrease in stride length of all four paws in A53T mice. One hundred hertz EA significantly increased its stride length in the left-fore, left-hind, and right-hind paw although not the right forelimb (Fig. 2d, e). The interlimb coordination measured by regularity index in A53T mice was reduced to $47.7 \%$ of control nTg mice. One hundred hertz EA recovered the regularity index by $84.6 \%$ during the course of the experiment (Fig. 2f). EA had no significant effect on all motor activities surveyed in $\mathrm{nTg}$ mice.

Noticeably, within 2 months of age, EA had a minimal impact on the motor impairments in movement distance, latency time, grip force, and Catwalk gait (data not shown). After 2 months of age, A53T mice showed an acceleration of the motor disability. At the same time, EA treatment had its maximal effects. The acceleration

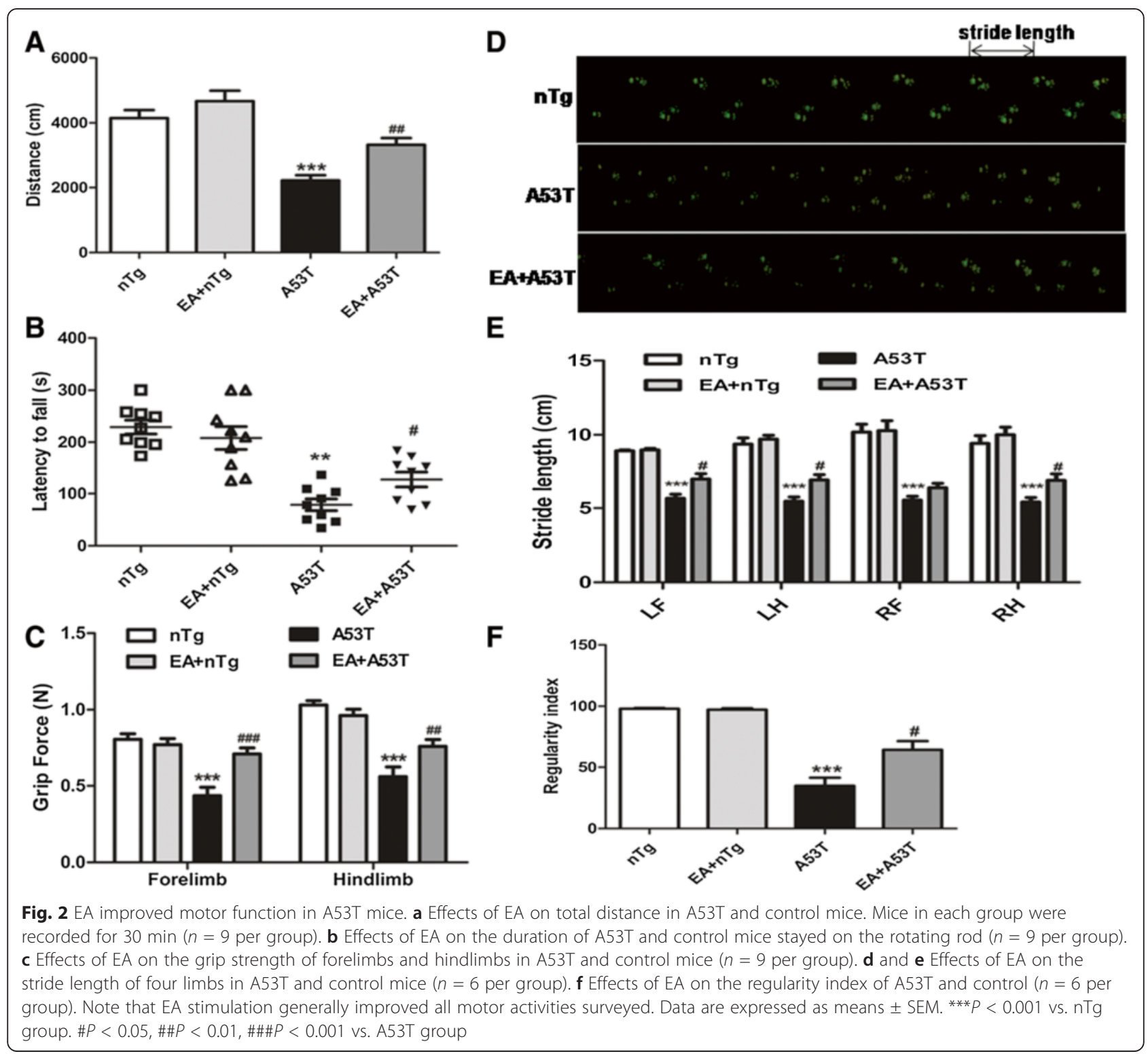


of the motor disability after 2 months might be due to substantial expression of $\alpha$-syn in astrocytes. EA by targeting overexpressed $\alpha$-syn could therefore exert its therapeutic effects (see below).

\section{EA alleviated astrocytic a-syn expression in A53T mice}

To observe the influence of EA on the aggregation of $\alpha$-syn, expression of A53T $\alpha$-syn in the midbrain and striatum was investigated in this study. Abundant expression of A53T $\alpha$-syn was seen in the GFAP-positive astrocytes as demonstrated by co-staining of $\alpha$-syn with GFAP in the SN (Fig. 3a). Western blots also showed that the level of exogenous $\alpha$-syn was gradually increased in the midbrain and striatum over different time points (1 month, 2 month, and symptomatic time) in A53T mice, while $\alpha$-syn immunoreactivity was absent in these regions in $\mathrm{nTg}$ control mice (Fig. 3b, c). After 4 weeks of $100 \mathrm{~Hz}$ EA treatment, the level of $\alpha$-syn proteins in the midbrain of A53T mice became significantly lower than that in untreated A53T mice (Fig. 4a). Similar results were observed in the striatum (Fig. 4b). In contrast to proteins, EA did not affect $\alpha$-syn mRNA expression in the midbrain (Fig. 4c) and striatum (Fig. 4d). In $n$ Tg mice with or without EA, no $\alpha$-syn expression at either protein or mRNA levels was observed. These data suggest that $100 \mathrm{~Hz}$ EA, while it did not affect A53T $\alpha$-syn transcription, downregulated $\alpha$-syn protein expression in the midbrain, and striatum of mutant mice.

\section{EA attenuated astrogliosis in A53T mice}

Astrocytic expression of A53T $\alpha$-syn appeared to disrupt multiple normal functions of astrocytes, resulting in severe astrogliosis throughout the brain and spinal cord [15]. We then assessed the rate of astrogliosis by analyzing expression of GFAP in A53T mice. Immunofluorescent staining revealed a larger number of GFAP-positive astrocytes in the SN of A53T mice (Fig. 5a). EA (100 $\mathrm{Hz}$ ) decreased the number of GFAP-immunoreactive astrocytes (Fig. 5a). Similarly, in immunoblot assays, EA effectively alleviated the excessive expression of GFAP in

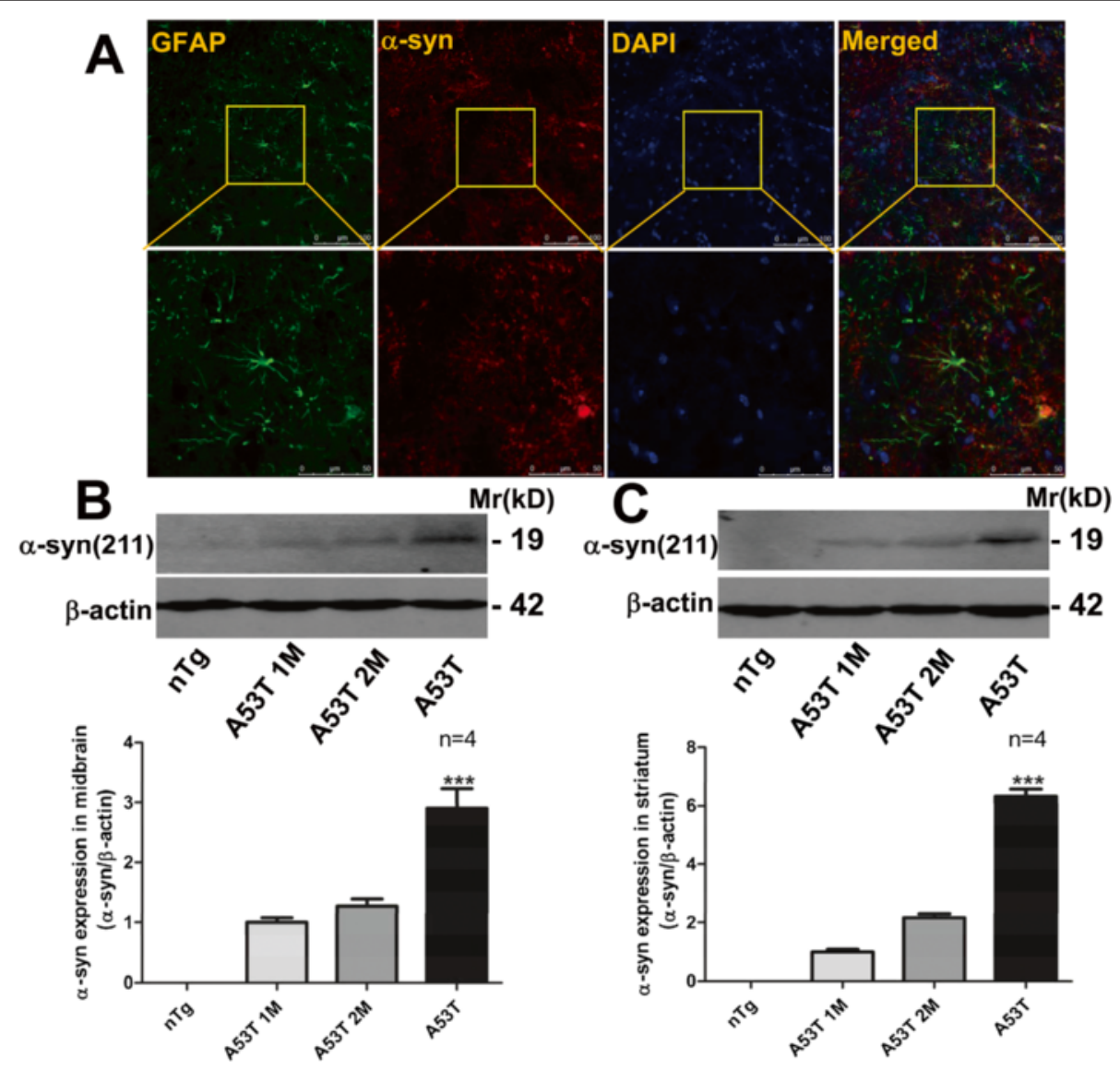

Fig. 3 Expression of a-syn in astrocytes in the SN and striatum of A53T mice. a Representative immunofluorescent photomicrographs showing a-syn (red) and GFAP (green) costaining in the SN of A53T mice. The a-syn immunoreactivity was restricted to GFAP-expressing astrocytes. $\mathbf{b}$ and c Immunoblot analysis of expression of a-syn proteins in the midbrain and striatum of A53T mice. Representative immunoblots are shown above the quantified data. A53T mice were sacrificed for subsequent immunoblot analysis at 1 or 2 months after the birth or at the paralysis development in the symptomatic A53T mice. Data are expressed as means \pm SEM ( $n=4$ per group). ${ }^{* * *} P<0.001$ vs. 1 or 2 months 


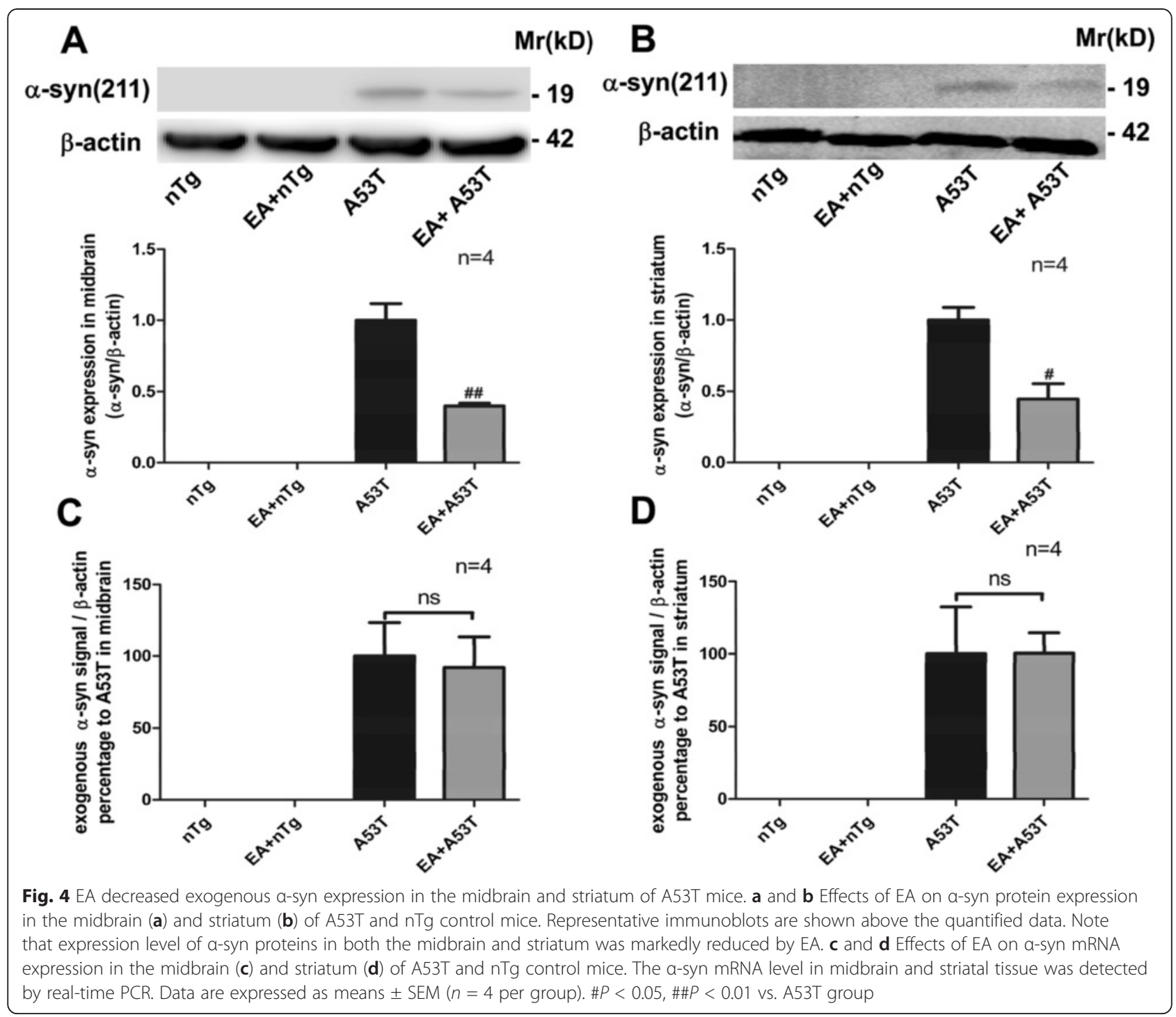

the SN of A53T mice (Fig. 5b). Of note, the level of GFAP in the EA-treated A53T mice still remained higher than that seen in control $\mathrm{nTg}$ mice. In addition, GFAP mRNA expression was probed using quantitative RT-PCR. We found that GFAP mRNA expression was elevated in the midbrain (Fig. 5c) and striatum (Fig. 5d) of A53T mice. However, unlike GFAP proteins, GFAP mRNA levels in these regions remained at a higher control level after EA treatment. The fact that EA limited the protein although no mRNA expression of GFAP in astrocytes of A53T mice indicates that EA may primarily inhibit reactive astrogliosis.

\section{EA alleviated microglial activation and inflammation responses in A53T mice}

Whether EA affects microglial activation and inflammatory responses was investigated in A53T mice. Activated microglia was observed in the SN of A53T mice, as evidenced by increased Iba- 1 immunoreactivity and by the appearance of microglia clusters (Fig. 6a). EA stimulation effectively reduced the intensity of Iba- 1 immunostaining and the clusters of activated microglia. Levels of inflammatory cytokines, such as TNF- $\alpha$ and IL- $1 \beta$ closely correlating with the presence of activated microglia, were quantified by ELISA in the SN and striatum. A53T mice exhibited a higher level of TNF- $\alpha$ expression in the midbrain and striatum, which was blocked by EA treatment (Fig. 6b). Similar results were seen for IL-1 $\beta$ expression in the striatum (Fig. 6c). Regarding IL-1 $\beta$ expression in the midbrain, an insignificant increase in IL-1 $\beta$ levels was observed in A53T mice after EA treatment. These results demonstrate the ability of EA to inhibit microglial activation and neuroinflammation induced by exogenous expression of A53T $\alpha$-syn in astrocytes. 

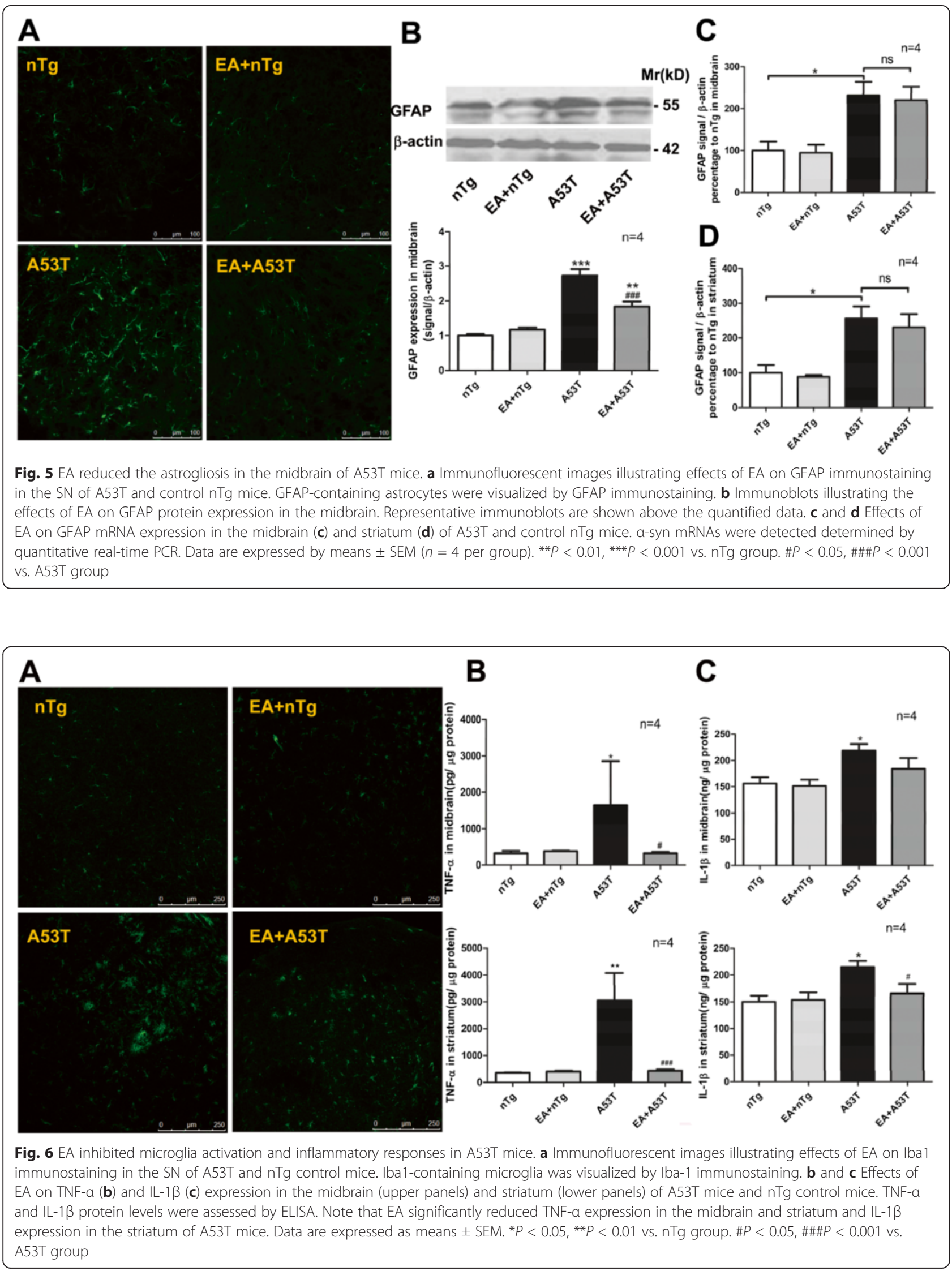
EA prevented dopaminergic neurodegeneration and loss of spinal motor neurons in A53T mice

To determine whether EA prevents neuronal loss in the midbrain of A53T mice, we monitored changes in the number of TH-positive dopaminergic neurons in the SNpc using an unbiased stereological method. As expected, the number of TH-positive neurons was decreased by $62.4 \%$ in the SNpc of A53T mice compared to nTg mice (Fig. 7a, b).
EA treatment significantly rescued the loss of neurons (Fig. 7a, b). A significant reduction of DA content (72.5\% of control) was seen in the striatum of A53T mice as detected by HPLC assays (Fig. 7c). EA stimulation partially reversed this reduction. In addition, reduced $\mathrm{TH}$ protein expression in the midbrain of mutant mice was partially recovered by EA (Fig. 7d). Thus, EA promotes survival of dopaminergic neurons in the midbrain of A53T mice.

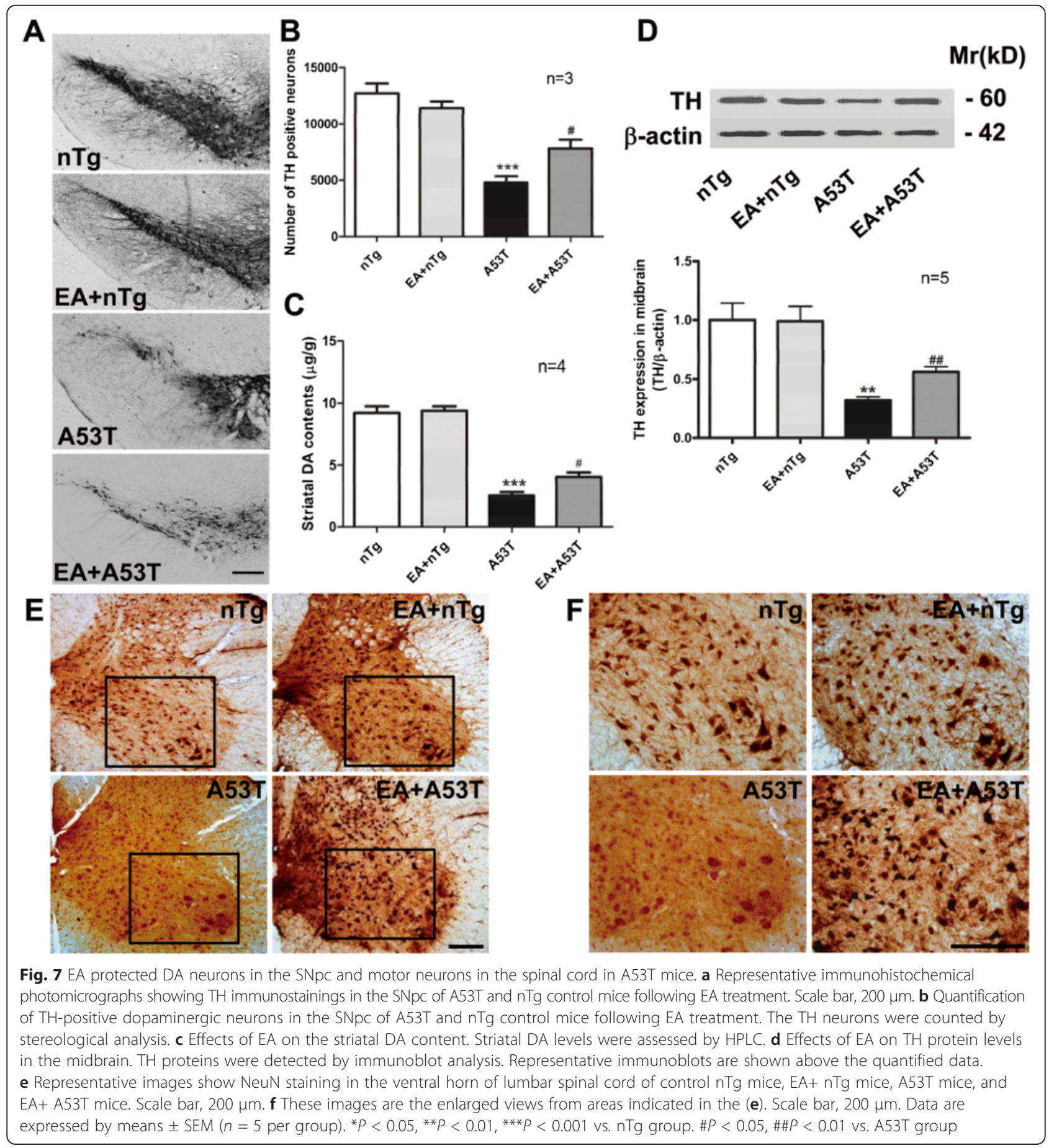


In addition, changes of neurons in the spinal cord were investigated by the immunostaining of NeuN. Cell bodies and dendrites of large motor neurons in the ventral horn were clearly stained in control mice. The density of immunostaining in motor neurons was reduced in both the cervical and lumber spinal cord of A53T mice compared to control mice (Fig. 7e, f). EA prevented the loss of these neurons in the spinal cord.

\section{EA modulated expression of Nrf2, HO-1, and GCLM proteins in A53T mice}

It is well known that Nrf2 suppresses neurodegeneration via a mechanism involving the inhibition of oxidative stress and gliosis. In A53T mice, Nrf2 protein levels were dramatically reduced in the midbrain and striatum compared to nTg mice (Fig. 8a, b). EA stimulation substantially increased Nrf2 proteins in the midbrain to a level higher than that in nTg control mice (Fig. 8a). EA also increased Nrf2 expression in the striatum (Fig. 8b). Meanwhile, EA markedly elevated the Nrf2 mRNA level in the midbrain of A53T mice compared to untreated-mice (Fig. 8c). Similarly, EA treatment significantly increased $\mathrm{HO}-1$ protein expression in the midbrain and striatum and GCLM expression in the midbrain (Fig. 8a, b). At the mRNA level, EA upregulated HO-1 and GCLM mRNA expression in the midbrain (Fig. 8c). These results indicate the ability of EA to upregulate expression of these proteins in both the midbrain and striatum of A53T mice.

\section{Discussion}

The principal finding of the present study was that high frequency EA stimulation alleviated motor impairments and delayed the progression of disease in A53T $\alpha$-syn transgenic mice. EA seems to be anti-inflammatory since it inhibited microglial activation, reduced cytokine production, and preserved the moderate astrogliosis in the

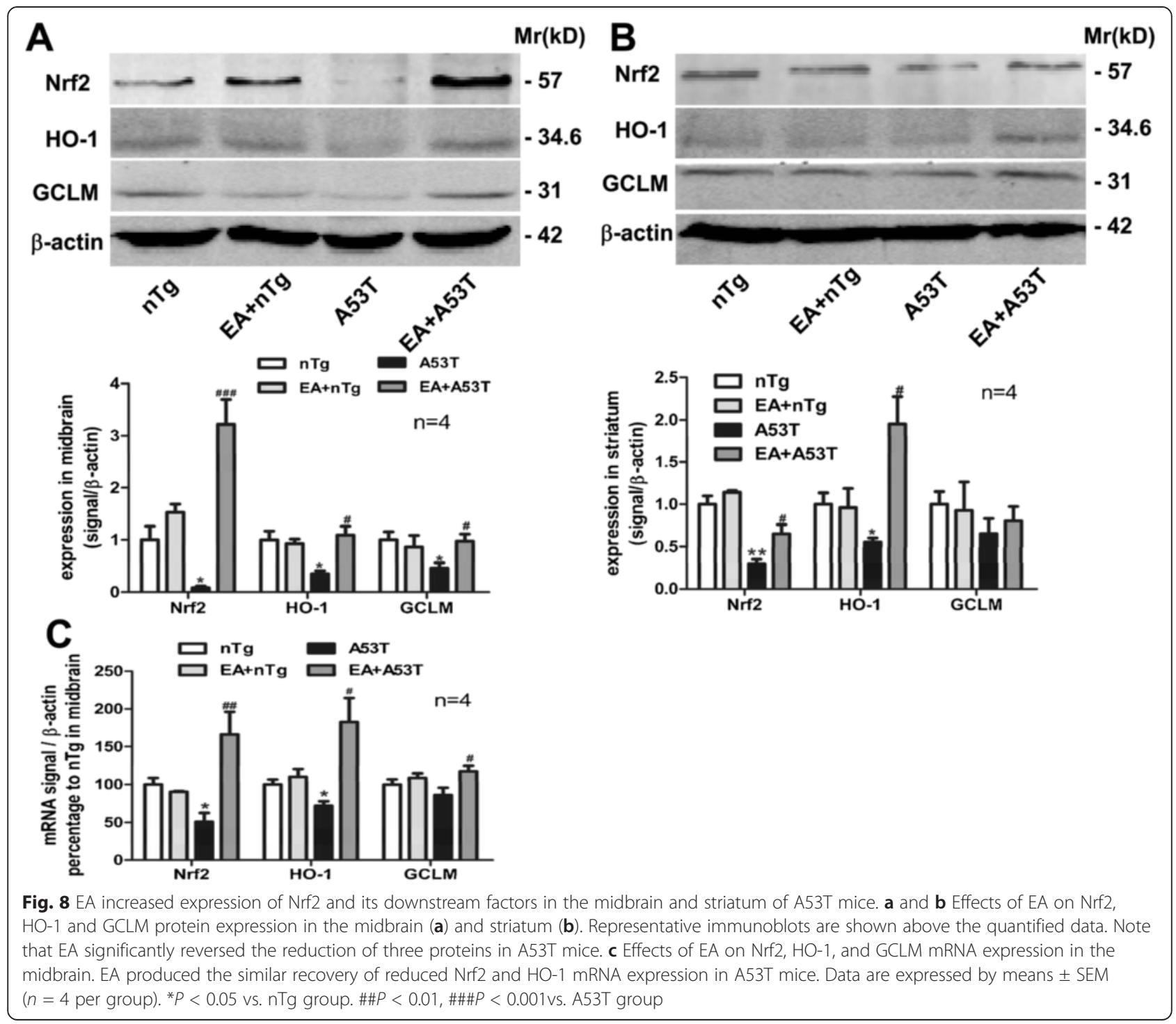


brain. EA also increased expression of Nrf2-dependent antioxidants and prevented the loss of midbrain dopaminergic neurons and motor neurons in the spinal cord. These effects establish EA as a useful tool for treating neuroinflammatory disorders.

\section{EA modulated a-syn pathology and alleviated motor disability in A53T mice}

According to Braak et al. [33], $\alpha$-syn deposition occurs at the earliest stage of PD without neuronal loss or clinical symptoms. The deposition takes place in both astrocytes and microglia [10], which eventually leads to the characteristic behavioral and pathological features of PD [34]. In transgenic mice, aggregated and truncated forms of $\alpha$-syn were significantly increased in the brain [15], which could impair the normal function of astrocytes and initiate the downstream pathogenic events [35]. In this study, we observed an accumulation of $\alpha$-syn proteins in the midbrain and striatum of symptomatic A53T mice. Interestingly, $\alpha$-syn expression was subject to the EA modulation. After prolonged EA stimulation, the amount of $\alpha$-syn proteins in the midbrain and striatum was reduced. While $\alpha$-syn protein levels were reduced, $\alpha$-syn mRNAs were not affected by EA. This seems to indicate that EA may primarily affect posttranscriptional steps, such as translation, aggregation, degradation, and/or propagation, to alter $\alpha$-syn protein expression. How EA regulates $\alpha$-syn expression is unclear. It has been found that EA increased ubiquitin $C$ terminal hydrolase-L1 (UCH-L1) and ubiquitin activating enzyme-1 (UBE1) expression in the SN, which led to a reduction of $\alpha$-syn expression and toxicity [36]. Thus, EA likely modulates $\alpha$-syn expression via a UCH-L1/ UBE1-dependent mechanism. In addition, apolipoprotein $\mathrm{D}$, a lipocalin involved in lipid metabolism, is constitutively expressed in astrocytes [37]. Due to structural similarities, lipocalins act as autocrine factors to interact with $\alpha$-syn and reduce reactive astrogliosis [37]. Thus, it is possible that EA could modulate the substances such as lipocalins to indirectly influence $\alpha$-syn and astrocytes.

The expression level of exogenous A53T $\alpha$-syn determines the onset and severity of behavioral abnormalities in these mutant mice. It has been found that the earlyonset paralysis occurred in A53T-E2 mice (the high expression of $\alpha$-syn in astrocytes), and however, there were no obvious behavior abnormalities in A53T-A8 mice (the low expression line) [15]. Thus, the improving effect of EA on motor behaviors may be derived from the regulation of $\alpha$-syn expression in A53T mice.

In A53T mice, excessive A53T $\alpha$-syn in astrocytes disrupted the normal function of astrocytes, leading to inflammation and microglial activation in the midbrain and spinal cord. Activated microglia then trigger the loss of neuron, which may contribute to the movement disability.
A recent study has found that conditional expression of tet-regulated A53T missense mutation $\alpha$-syn in the midbrain dopaminergic neurons caused key motor and pathological phenotypes of PD [38], although not paralysis. This indicates that the paralysis phenotype results from the loss of motor neurons in the spinal cord. In this study, EA was effective in alleviating both PD-like motor behaviors and paralysis in A53T mice, which corresponds well with the EA effect in preventing the loss of dopaminergic neurons in the midbrain and motor neurons in the spinal cord. Taken together, EA reverses the loss of neurons in the midbrain and spinal cord to improve respective motor activities.

\section{Roles of reactive astrogliosis in PD and EA effects}

Severe reactive astrogliosis interferes with the normal function of astrocytes that is critical for maintaining the integrity of blood-brain barrier (BBB) and for regulating the homeostasis of glutamate transporters [15]. While severe astrogliosis is disadvantageous, reactive astrocytes also play a compensatory role. This was evidenced by the finding that selective and conditional ablation of reactive astrocytes caused greater neuronal and oligodendrocyte degeneration, greater inflammatory responses, less recovery of the $\mathrm{BBB}$, and more severe functional deficits in a spinal cord injury transgenic mouse [39]. Moreover, reactive astrocytes promoted dopaminergic neuronal recovery [40]. In our studies, A53T mice showed severe astrogliosis as evidenced by a substantial increase in the number of GFAP-positive astrocytes and GFAP protein overexpression in the SN. EA was able to partially reduce astrogliosis. However, it is important to note that EA did not completely abolish reactive astrogliosis. As a matter of fact, a moderate and significant degree of reactive astrogliosis remained following EA stimulation. Thus, EA seems to tune down the excessive reactive astrogliosis and at the same time preserve moderate reactive astrogliosis. This fine adjustment is useful to preserve a certain amount of reactive astrogliosis beneficial for neuronal survival.

The functional role of reactive astrocytes in regulating neuroinflammation is noteworthy $[41,10]$. In A53T mice, reactive astrocytes are major cells that recruit and trigger the activation of microglia and increase expression of proinflammatory cytokines such as TNF- $\alpha$ and IL-1 $\beta$ in the midbrain and striatum. By activating microglia and releasing cytokines, astrocytes are linked to the loss of dopamine neurons and motor symptoms of PD. In support of this, we found that the number of activated microglia was enhanced in the SN of A53T mice. Parallel increases in pro-inflammatory cytokines (TNF- $\alpha$ and IL-1 $\beta$ ) were also observed in the midbrain and striatum. Meanwhile, EA inhibited microglial activation and inflammatory cytokine expression. These results suggest that EA may exert its alleviating effects on PD symptoms through a 
mechanism involving the inhibition of microglia activation and microglia-mediated inflammatory reactions.

Of note, microglia activation is classified into two major phenotypes: detrimental M1 (classical activation) and beneficial M2 (alternative activation) phenotypes [42]. Astrocytic expression of A53T certainly evoked M1 microglial activation at the symptomatic stage. EA effectively reduced the activation of microglia and expression of M1related inflammatory cytokines, such as TNF- $\alpha$ and IL-1 $\beta$. Little is known about the activation of the M2 phenotype in the PD pathogenesis [43]. Recently, an epigenetic study has suggested that histone H3K27me3 demethylase Jmjd3 may facilitate the switch of microglia phenotypes from M2 to M1 polarization [44]. Therefore, it will be interesting to know whether EA helps switch M1/M2 phenotypes in the future studies.

\section{Nrf2 signal pathways in PD and EA effects}

An important anti-oxidant and anti-inflammatory mechanism involves the Nrf2-ARE pathway. Selective Nrf2 expression in astrocytes is sufficient to prevent the 1methyl-4-phenyl-1, 2, 3, 6-tetrahydropyridine-induced loss of dopaminergic neurons [40]. In response to exogenously added $\alpha$-syn, $\mathrm{Nrf2}^{-/-}$mice showed an exacerbated loss of nigral dopaminergic neurons and increased neuroinflammation and gliosis at the early PD stage [28]. Therefore, the activation of the Nrf2-ARE pathway is of a neuroprotective nature [45]. Consistent with this, Nrf2 modulated microglial activation to produce anti-inflammatory responses [46]. In contrast, impaired Nrf2 responses correlated with a shift in the microglial activation profile, towards an increased production of proinflammatory markers (IL-6, IL-1 $\beta$, iNOS, and NF- $\mathrm{kB}$ ). The relevance of Nrf2-regulated genes in inflammation has also been identified in postmortem biopsies of PD patients. An increase in HO-1 expression in glial cells has been revealed, which however was insufficient to elicit an effective protection of SN neurons [45]. In this study, we observed lower expression of Nrf2 and its downstream molecules (HO-1 and GCLM) in the midbrain and striatum of A53T mice. EA was able to upregulate expression of these proteins. Of note, the Nrf2 protection is not restricted to $\alpha$-syn toxicity. In fact, Nrf2 plays a role in overall neuronal degeneration seen in oxidative stress, environmental toxins, proteinopathy, and inflammation [45]. Thus, Nrf2 represents a common site for developing pharmacotherapies to treat a wide range of neurodegenerative diseases, including PD, Alzheimer's disease, and Huntington's disease.

\section{Potential anti-inflammation pathways of EA}

Neural mechanisms underlying the EA effect have long been investigated $[24,47,25]$. Recent studies suggest a neural-immune model. EA is typically applied to the deep tissue enriched with sensory innervations [48].
Sensory signals induced by EA stimulation alter the immune system to dynamically control immunity activity [49]. Indeed, EA stimulation at the ST36 Zusanli acupoint decreased the lipopolysaccharide-induced inflammatory cytokines, including TNF, monocyte chemotactic protein-1, IL-6, and interferon- $\gamma$ [24]. However, the antiinflammatory action of EA was abolished by surgical sectioning of the sciatic nerve. Conversely, direct stimulation of the sciatic nerve mimicked the anti-inflammatory effect of EA [24]. Thus, it appears that EA stimulation of the acupoints on the hind-limb acts as an effective vagal stimulation, which can control inflammation. However, the exact anatomical pathways connecting EA signals to the immune system are poorly understood [50]. Future studies will explore those pathways in details.

\section{Conclusions}

Our results demonstrate that EA effectively increased survival of A53T mice, decreased non-neuronal expression of $\alpha$-syn, and inhibited inflammatory responses. Meanwhile, EA preserved the moderate astrogliosis, protected the dopaminergic neurons, and alleviated motor impairments. These data indicate a causative relationship between glial $\alpha$-syn-mediated inflammation responses and chronic dopaminergic neurodegeneration. Moreover, EA by inhibiting degenerative processes at multiple levels has potential to be a useful tool for treating neurodegenerative diseases.

\section{Abbreviations}

a-syn: a-synuclein; ANOVA: analysis of variance; ARE: Antioxidant response element; BBB: Blood-brain barrier; DAPI: 4',6-diamidino-2-phenylindole; DOX: Doxycycline; EA: Electroacupuncture; ELISA: Enzyme-linked immunosorbent assay; GCLM: Glutamate-cysteine ligase modifier subunits; GFAP: Glial fibrillary acidic protein; HO-1: Heme oxygenase-1; Iba1: Ionized calcium-binding adaptor molecule-1; IL-1 $\beta$ : Interleukin-1 $\beta$; IL-6: Interleukin-6; NeuN: Neuronal nuclei; Nrf2: Nuclear factor E2-related factor 2; PCR: Polymerase chain reaction; PD: Parkinson's disease; PBS: Phosphate buffered saline; SN: Substantia nigra; SNpc: Substantia nigra pars compacta; tetO: Tetracycline operator; TH: Tyrosine hydroxylase; TNF-a: Tumor necrosis factor-a; UBE1: Ubiquitin activating enzyme-1; UCH-L1: Ubiquitin C terminal hydrolase-L1.

\section{Competing interests}

The authors declare that they have no competing interests.

\section{Authors' contributions}

$J \mathrm{D}, J \mathrm{~J}$, and XW participated in the research design, data analysis, and wrote or contributed to the writing of the manuscript. EL and XG participated in the molecular studies and the immunoassays. WZ and JW participated in the animal behavior tests. JY participated in the design of the study and performed the statistical analysis. XL participated in the study design and helped to draft the manuscript. All authors read and approved the final version of the manuscript.

\section{Acknowledgements}

This study was supported by the National Basic Research Program of China (2011CB504100), the National Natural Science Foundation of China (81072858, 81473770, 81030062), Beijing Natural Science Foundation (7152021), the Importation and Development of High-Caliber Talents Project of Beijing Municipal Institutions (CIT\&TCD201304185), the Project of Construction of Innovative Teams and Teacher Career Development for 
Universities and Colleges under the Beijing Municipality (IDHT20140514), and the Seed Grant of International Alliance of Translational Neuroscience (PXM2014_014226_000015). We express our sincere thanks to Wang Qiang (University of Missouri-Kansas City) for his critical readings of the manuscript, to Dr. Huaibin Cai (National Institute of Health) for providing the TetO-A53T and GFAP-tTA mice and important suggestions in this study.

\section{Author details}

'Departments of Neurobiology and Physiology, Key Laboratory for Neurodegenerative Disorders of the Ministry of Education, Beijing Key Laboratory for Parkinson's Disease, Capital Medical University; Beijing Institute for Brain Disorders, Beijing 100069, China. ${ }^{2}$ Department of Neurology and Neurological Sciences, Stanford University, Stanford, CA 94305, USA.

\section{Received: 8 January 2015 Accepted: 16 April 2015}

\section{Published online: 28 May 2015}

\section{References}

1. Lee HJ, Suk JE, Bae EJ, Lee JH, Paik SR, Lee SJ. Assembly-dependent endocytosis and clearance of extracellular alpha-synuclein. Int J Biochem Cell Biol. 2008;40:1835-49.

2. Tokuda T, Salem SA, Allsop D, Mizuno T, Nakagawa M, Qureshi MM, et al Decreased alpha-synuclein in cerebrospinal fluid of aged individuals and subjects with Parkinson's disease. Biochem Biophys Res Commun. 2006;349:162-6.

3. El-Agnaf OM, Salem SA, Paleologou KE, Cooper L, Fullwood NJ, Gibson MJ, et al. Alpha-synuclein implicated in Parkinson's disease is present in extracellular biological fluids, including human plasma. FASEB J. 2003;17:1945-7.

4. Jang A, Lee HJ, Suk JE, Jung JW, Kim KP, Lee SJ. Non-classical exocytosis of alpha-synuclein is sensitive to folding states and promoted under stress conditions. J Neurochem. 2010;113:1263-74.

5. Lee HJ, Patel S, Lee SJ. Intravesicular localization and exocytosis of alpha-synuclein and its aggregates. J Neurosci. 2005;25:6016-24

6. Lee SJ, Desplats P, Sigurdson C, Tsigelny I, Masliah E. Cell-to-cell transmission of non-prion protein aggregates. Nat Rev Neurol. 2010;6:702-6.

7. Kordower JH, Chu Y, Hauser RA, Freeman TB, Olanow CW. Lewy body-like pathology in long-term embryonic nigral transplants in Parkinson's disease. Nat Med. 2008;14:504-6.

8. Lee HJ, Suk JE, Bae EJ, Lee SJ. Clearance and deposition of extracellular alpha-synuclein aggregates in microglia. Biochem Biophys Res Commun. 2008;372:423-8

9. Hansen C, Angot E, Bergstrom AL, Steiner JA, Pieri L, Paul G, et al. alphaSynuclein propagates from mouse brain to grafted dopaminergic neurons and seeds aggregation in cultured human cells. J Clin Invest. 2011;121:715-25.

10. Halliday GM, Stevens CH. Glia: initiators and progressors of pathology in Parkinson's disease. Mov Disord. 2011;26:6-17.

11. Braak H, Sastre M, Del Tredici K. Development of alpha-synuclein immunoreactive astrocytes in the forebrain parallels stages of intraneuronal pathology in sporadic Parkinson's disease. Acta Neuropathol. 2007;114:231-41.

12. Beyer K, Ariza A. Protein aggregation mechanisms in synucleinopathies: commonalities and differences. J Neuropathol Exp Neurol. 2007;66:965-74.

13. Croisier E, Graeber MB. Glial degeneration and reactive gliosis in alphasynucleinopathies: the emerging concept of primary gliodegeneration Acta Neuropathol. 2006:112:517-30.

14. Wakabayashi K, Hayashi S, Yoshimoto M, Kudo H, Takahashi H. NACP/alphasynuclein-positive filamentous inclusions in astrocytes and oligodendrocytes of Parkinson's disease brains. Acta Neuropathol. 2000;99:14-20.

15. Gu XL, Long CX, Sun L, Xie C, Lin X, Cai H. Astrocytic expression of Parkinson's disease-related A53T alpha-synuclein causes neurodegeneration in mice. Mol Brain. 2010;3:12.

16. Lee HJ, Suk JE, Patrick C, Bae EJ, Cho JH, Rho S, et al. Direct transfer of alpha-synuclein from neuron to astroglia causes inflammatory responses in synucleinopathies. J Biol Chem. 2010;285:9262-72.

17. Hirsch EC, Hunot S. Neuroinflammation in Parkinson's disease: a target for neuroprotection? Lancet Neurol. 2009;8:382-97.

18. Vickers AJ, Cronin AM, Maschino AC, Lewith G, MacPherson H, Foster NE, et al. Acupuncture for chronic pain: individual patient data meta-analysis. Arch Intern Med. 2012;172:1444-53.
19. Wu HM, Tang JL, Lin XP, Lau J, Leung PC, Woo J, et al. Acupuncture for stroke rehabilitation. Cochrane Database Syst Rev. 2006: Art. No.: CD004131. doi:10.1002/14651858.CD004131.pub2.

20. Yang EJ, Jiang JH, Lee SM, Hwang HS, Lee MS, Choi SM. Electroacupuncture reduces neuroinflammatory responses in symptomatic amyotrophic lateral sclerosis model. J Neuroimmunol. 2010;223:84-91.

21. Chuang CM, Hsieh CL, Li TC, Lin JG. Acupuncture stimulation at Baihui acupoint reduced cerebral infarct and increased dopamine levels in chronic cerebral hypoperfusion and ischemia-reperfusion injured Sprague-Dawley rats. Am J Chin Med. 2007;35:779-91.

22. Kang JM, Park HJ, Choi YG, Choe IH, Park JH, Kim YS, et al. Acupuncture inhibits microglial activation and inflammatory events in the MPTP-induced mouse model. Brain Res. 2007:1131:211-9.

23. Liu XY, Zhou HF, Pan YL, Liang XB, Niu DB, Xue B, et al. Electro-acupuncture stimulation protects dopaminergic neurons from inflammation-mediated damage in medial forebrain bundle-transected rats. Exp Neurol. 2004;189:189-96

24. Torres-Rosas R, Yehia G, Pena G, Mishra P, del Rocio T-BM, Moreno-Eutimio $M A$, et al. Dopamine mediates vagal modulation of the immune system by electroacupuncture. Nat Med. 2014;20:291-5

25. Goldman N, Chen M, Fujita T, Xu Q, Peng W, Liu W, et al. Adenosine A1 receptors mediate local anti-nociceptive effects of acupuncture. Nat Neurosci. 2010;13:883-8.

26. Kavoussi B, Ross BE. The neuroimmune basis of anti-inflammatory acupuncture. Integr Cancer Ther. 2007;6:251-7.

27. Zhang RX, Lao L, Wang X, Fan A, Wang L, Ren K, et al. Electroacupuncture attenuates inflammation in a rat model. J Altern Complement Med. 2005;11:135-42.

28. Chen PC, Vargas MR, Pani AK, Smeyne RJ, Johnson DA, Kan YW, et al. Nrf2mediated neuroprotection in the MPTP mouse model of Parkinson's disease: critical role for the astrocyte. Proc Natl Acad Sci U S A. 2009;106:2933-8.

29. Wang H, Pan Y, Xue B, Wang X, Zhao F, Jia J, et al. The antioxidative effect of electro-acupuncture in a mouse model of Parkinson's disease. PLoS One. 2011;6, e19790.

30. Choi YG, Yeo S, Hong YM, Lim S. Neuroprotective changes of striatal degeneration-related gene expression by acupuncture in an MPTP mouse model of Parkinsonism: microarray analysis. Cell Mol Neurobiol. 2011;31:377-91.

31. Masocha W, Parvathy SS. Assessment of weight bearing changes and pharmacological antinociception in mice with LPS-induced monoarthritis using the Catwalk gait analysis system. Life Sci. 2009;85:462-9.

32. Paxinos G, Franklin KBJ. The Mouse Brain in Stereotaxic Coordinates. 2nd ed. San Diego: Academic; 2001

33. Braak H, Del Tredici K, Rub U, de Vos RA, Jansen Steur EN, Braak E. Staging of brain pathology related to sporadic Parkinson's disease. Neurobiol Aging. 2003;24:197-211

34. Luk KC, Kehm V, Carroll J, Zhang B, O'Brien P, Trojanowski JQ, et al. Pathological alpha-synuclein transmission initiates Parkinson-like neurodegeneration in nontransgenic mice. Science. 2012;338:949-53.

35. Li W, West N, Colla E, Pletnikova O, Troncoso JC, Marsh L, et al. Aggregation promoting C-terminal truncation of alpha-synuclein is a normal cellular process and is enhanced by the familial Parkinson's disease-linked mutations. Proc Natl Acad Sci U S A. 2005;102:2162-7.

36. Wang YC, He F, Ma J, Zhou D, Liang Y, Gong YX. Impacts of electroacupuncture on ubiquitin-proteasome system in rats with Parkinson's disease. Zhongguo Zhen Jiu. 2013;33:725-9.

37. Navarro A, Del Valle E, Tolivia J. Differential expression of apolipoprotein d in human astroglial and oligodendroglial cells. J Histochem Cytochem. 2004;52:1031-6.

38. Lin X, Parisiadou L, Sgobio C, Liu G, Yu J, Sun L, et al. Conditional expression of Parkinson's disease-related mutant alpha-synuclein in the midbrain dopaminergic neurons causes progressive neurodegeneration and degradation of transcription factor nuclear receptor related 1. J Neurosci. 2012;32:9248-64

39. Faulkner JR, Herrmann JE, Woo MJ, Tansey KE, Doan NB, Sofroniew MV. Reactive astrocytes protect tissue and preserve function after spinal cord injury. J Neurosci. 2004;24:2143-55.

40. Episcopo FL, Tirolo C, Testa N, Caniglia S, Morale MC, Marchetti B. Reactive astrocytes are key players in nigrostriatal dopaminergic neurorepair in the MPTP mouse model of Parkinson's disease: focus on endogenous neurorestoration. Curr Aging Sci. 2013;6:45-55. 
41. Lee HJ, Kim C, Lee SJ. Alpha-synuclein stimulation of astrocytes: potential role for neuroinflammation and neuroprotection. Oxid Med Cell Longev. 2010;3:283-7.

42. Cherry JD, Olschowka JA, O'Banion MK. Neuroinflammation and M2 microglia: the good, the bad, and the inflamed. J Neuroinflammation. 2014;11:98.

43. Tang Y, Le W. Differential Roles of M1 and M2 Microglia in Neurodegenerative Diseases. Mol Neurobiol. in press. doi: 10.1007/s12035-014-9070-5.

44. Tang Y, Li T, Li J, Yang J, Liu H, Zhang XJ, et al. Jmjd3 is essential for the epigenetic modulation of microglia phenotypes in the immune pathogenesis of Parkinson's disease. Cell Death Differ. 2014;21:369-80.

45. Lastres-Becker I, Ulusoy A, Innamorato NG, Sahin G, Rabano A, Kirik D, et al. alpha-Synuclein expression and Nrf2 deficiency cooperate to aggravate protein aggregation, neuronal death and inflammation in early-stage Parkinson's disease. Hum Mol Genet. 2012;21:3173-92.

46. Innamorato NG, Rojo Al, Garcia-Yague AJ, Yamamoto M, de Ceballos ML, Cuadrado A. The transcription factor Nrf2 is a therapeutic target against brain inflammation. J Immunol. 2008;181:680-9.

47. Ernst E, Lee MS, Choi TY. Acupuncture: does it alleviate pain and are there serious risks? A review of reviews. Pain. 2011;152:755-64.

48. Zhao ZQ. Neural mechanism underlying acupuncture analgesia. Prog Neurobiol. 2008;85:355-75.

49. Chavan SS, Tracey KJ. Regulating innate immunity with dopamine and electroacupuncture. Nat Med. 2014;20:239-41.

50. Asakawa T, Fang H, Hong Z, Sugiyama K, Nozaki T, Namba H. Peripheral stimulation in treating Parkinson's disease: is it a realistic idea or a romantic whimsicality? Intractable Rare Dis Res. 2012;1:144-50.

\section{Submit your next manuscript to BioMed Central and take full advantage of:}

- Convenient online submission

- Thorough peer review

- No space constraints or color figure charges

- Immediate publication on acceptance

- Inclusion in PubMed, CAS, Scopus and Google Scholar

- Research which is freely available for redistribution 